\title{
Indikatorenarbeit
}

\author{
Kontextneutralisierende und kontextoffene Strategien \\ in der Analyse komplexer Probleme
}

\author{
Stefan Böschen, Human Technology Center (HumTec) RWTH Aachen University, Theaterplatz 14, 52062 Aachen \\ (stefan.boeschen@humtec.rwth-aachen.de), (1) orcid.org/0000-0003-0519-5030 \\ Mahshid Sotoudeh, Institut für Technikfolgen-Abschätzung (ITA-ÖAW) (Mahshid.Sotoudeh@oeaw.ac.at) \\ Volker Stelzer, Institut für Technikfolgenabschätzung und Systemanalyse (ITAS), Karlsruher Institut für Technologie (KIT) (volker.stelzer@kit.edu)
}

Die Analyse komplexer Probleme, unter anderem mittels Indikatoren, gehört zum Alltag der Technikfolgenabschätzung (TA). Indikatorenarbeit verdeutlicht, dass die Auswahl von Indikatoren, trotz genereller Regeln, auf akteursabhängigen Entscheidungen beruht. Die These des Artikels lautet, dass TA kontextneutralisierende wie kontextoffene Analysestrategien nutzt. Die Analyse von Indikatorenarbeit an einem Beispiel im Feld der Nachhaltigkeitsanalyse zeigt, wie die Spannung zwischen diesen beiden Analysestrategien in der Indikatorenarbeit durch Verfahrensregeln transparent zu machen ist. Normative Transparenz kann durch reflektierte Indikatorenarbeit in der TA sichergestellt werden.

\section{Indicator work}

Context-neutralizing and context-open strategies in the analysis of complex problems

The analysis of complex problems, e. g., using indicators, is part of technology assessment (TA) practice. The term "indicator work" illustrates that the selection of indicators is, besides general rules, based on individual decisions. The hypothesis is that TA uses two analytical strategies: context-neutralizing and context-open variants. Looking at an example of sustainability analysis in TA, we will show how the tension between the two strategies can be made transparent by procedural norms in indicator work. Normative transparency can be ensured by reflective indicator work in TA.

Keywords: indicators, sustainable development, options for action, valuation, complex problems

This is an article distributed under the terms of the Creative Commons Attribution License CCBY 4.0 (https://creativecommons.org/licenses/by/4.0/)

https://doi.org/10.14512/tatup.28.1.45

Submitted: 22. 10.2018. Peer reviewed. Accepted: 13.02.2019

\section{Einleitung}

Die Analyse komplexer Probleme gehört zum Alltagsgeschäft von Technikfolgenabschätzung (TA). Jede für TA bedeutsame Frage bezieht sich auf Probleme, für die gesellschaftlicher Entscheidungsbedarf schon besteht bzw. entstehen kann. Komplexe Probleme zu analysieren, muss jedoch methodologisch als ein anspruchsvolles Alltagsgeschäft angesehen werden. Denn die damit verbundene Auswahl und Spezifizierung von Indikatoren (Indikatorenarbeit) verlangt eine reflektierte Strategie, um komplexen Problemen aufgrund normativer Voreinstellungen nicht eine unzutreffende Gestalt zu geben. Dass hier eine für die TA herausfordernde Reflexionsaufgabe besteht, zeigt sich an der typischerweise in der TA genutzten Unterscheidung zwischen Technikfolgenanalyse und Technikbewertung. Denn diese suggeriert eine Trennung von zwei Arbeitsschritten innerhalb der TA, deren Voraussetzung nicht immer gegeben, aber gleichwohl normativ relevant ist. Dagegen vertreten wir die These, dass in der TA zwei kontrastierende Strategien der Analyse von komplexen Problemen angewandt werden: kontextneutralisierende im Gegensatz zu kontextoffenen.

Vor diesem Hintergrund gliedert sich der Aufsatz in vier Teile. Erstens werden kontextneutralisierende und kontextoffene Formen der TA-Analyse idealtypisch aufgezeigt und auf dieser Grundlage Indikatorenarbeit definiert. Zweitens werden Problemkreise der Indikatorenarbeit herausgestellt. In einem dritten Schritt stellen wir die konkreten Herausforderungen der Indikatorenarbeit an einem TA-Projekt vor, die sich in der Fülle der Auswahlentscheidungen zeigt. Anschließend werden die Einsichten ausgewertet und ein Ausblick auf künftige Perspektiven gegeben.

\section{Analysestrategien in der TA: kontextneutralisierend - kontextoffen}

Für vielschichtige Sachverhalte der Technologie- und Innovationsentwicklung erzeugt TA entscheidungsrelevantes Wissen. Um nicht unter den Verdacht politischer Vorteilnahme oder des 
Verfolgens von Partialinteressen zu geraten, sollte dieses Wissen in besonderer Weise wissenschaftliche Rationalität verkörpern (Grunwald 1999). Das gelang am überzeugendsten mittels möglichst standardisierter Wissensmodelle und Quantifizierung der Nebenfolgen durch ausgesuchte Verfahren, wie z.B. die Kosten-Nutzen-Analyse (Bröchler et al. 1999). Diese bilden auch weiterhin das methodische Rückgrat der TA neben neuerer Ansätze der konstruktiven TA, der partizipativen TA oder der antizipativen TA.

So kamen mit der Zeit andere Fragestellungen hinzu, die weniger ,technikinduziert“ als vielmehr ,problembezogen“ waren (Bechmann 1993). Die Debatte um Nachhaltige Entwicklung ist die Integration von Umweltkomplexität von großer Bedeutung ist und enge Systemgrenzen aufgebrochen werden sollen, etwa in der Medizin, der Pflege, der Bildung oder auch der Seelsorge. Deshalb kann es ,,in kontextoffenen Operationen nicht mehr allein darum gehen, eine systeminterne Operation durchzuführen und alles weitere der Umwelt zu überlassen, vielmehr muss die Bearbeitung der Umwelt selbst noch in die interne Operation aufgenommen werden“ (ebd.). Entscheidend ist hierbei, dass trotz der Schaffung eines bearbeitungsfähigen Binnenbereichs, der Komplexität zu reduzieren erlaubt, ,die aufgebauten Grenzen zwischen Innen und Außen im betrachteten System immer wieder durchbrochen werden“ (ebd.). Die Differenz zwischen

\section{Die Komplexität von Problemen spiegelt sich in den Schwierigkeiten, kontextneutralisierende und kontextoffene Analysestrategien sinnvoll zu konfigurieren.}

hierbei der paradigmatische Fall. Die Analyse von Nachhaltigkeit bedarf methodischer Verfahren, die anderen Rationalitätsmaßstäben folgen. Dieser Wechsel lässt sich mit einer Unterscheidung beschreiben, die zur Charakterisierung zwischen kontextneutralisierenden und kontextoffenen Formen der Risikokommunikation eingeführt wurde (Giegel 1993). Obgleich diese Unterscheidung einer anderen Debatte entstammt, stellt sie gleichwohl ein formtheoretisches Argument dar, das für andere Problemstellungen fruchtbar gemacht werden kann, so auch für Analysestrategien komplexer Probleme innerhalb der problemorientierten Forschung.

Die erste Form der Risikokommunikation zeichne sich, so Giegel, dadurch aus, dass sie einer Operationsweise ähnlich der von ausdifferenzierten Teilsystemen folge: Kontextneutralisierende Operationen ,sind dadurch gekennzeichnet, dass sie eine strikte Grenze ziehen zwischen einem Innenbereich, in dem Komplexität drastisch reduziert, und einem Außenbereich, den man in seiner Überkomplexität nicht versteht und schon gar nicht praktisch beherrscht" (Giegel 1993, S. 107). Jedoch fordert dieser Gewinn an Rationalisierung im Binnenbereich eine besondere Stabilität von sozialen und naturalen Faktoren. Denn ganz offensichtlich können solche Operationen, wie sie etwa in großen Kraftwerken realisiert sind, die für sie erforderliche sozio-materielle Umwelt (also etwa: erforderliche Standards, Infrastrukturen, Humankapital, gesetzliche Grundlagen des Betreibens u. v. m.) nicht selbst herstellen. Kontextneutralisierende Operationen ignorieren auf der Grundlage solcher Voraussetzungen Umweltkomplexität.

Im Gegensatz dazu bleibe bei kontextoffenen Operationen „der Versuch einer strikten Grenzziehung zwischen systemintern geregelter und systemexterner lebensweltlicher Kommunikation [...] in sich widersprüchlich“ (ebd., S. 108). Diese Form von Operationen finden wir gerade in solchen Bereichen, in denen diesen beiden Operationsweisen besteht also nicht in der Schaffung von komplexitätsentlasteten Binnenbereichen, sondern in der Sensibilität bezüglich des Umgangs mit den dadurch zugleich geschaffenen Grenzen.

In der TA finden wir die Anwendung beider Operationsformen als Analysestrategien. TA nutzt kontextneutralisierende Operationen in den Fällen, in denen schon eine klare Systemdefinition für das zu thematisierende Problem vorliegt. Kontextoffene Operationen sind dann hilfreich für TA-Analysen, wenn die Konturen des Problems nicht abschließend definiert und gerade die Exploration des Problems Teil der Fragestellung für TA sind. Eine gegenstandsadäquate Auswahl von Analysestrategien stellt prinzipiell eine Herausforderung für TA dar. Eine Tendenz zu kontextneutralisierenden Analysestrategien ist zu konstatieren. Die Komplexität von Problemen spiegelt sich in den Schwierigkeiten, kontextneutralisierende und kontextoffene Analysestrategien sinnvoll zu konfigurieren. Begrifflich kann Indikatorenarbeit als die Auswahl- und Konfigurationstätigkeit von Indikatoren im Spannungsfeld dieser beiden Analysestrategien verstanden werden.

\section{Analysestrategien und Indikatorenarbeit in der TA}

Indikatoren repräsentieren Effekt-bezogene Wirklichkeitsausschnitte aus zu analysierenden Problemfeldern. Im Gegensatz dazu lassen sich Kriterien in der Weise definieren, dass sie vor dem Hintergrund bestimmter Werthaltungen und Interessen Indikatoren valuieren und für die Charakterisierung eines Problems als relevant positionieren (Boavida und Böschen 2015, S. 38). Die Auswahl und der Zuschnitt von Indikatoren werden umso aufwändiger und schwieriger je komplexer sich das Pro- 
blem darstellt. Nehmen wir ein Beispiel aus dem Bereich der Mobilität. Die Nutzung von Pkws hat nach dem zweiten Weltkrieg nicht nur zugenommen, sondern zugleich zeigen sich relevante Engführungen in der Analyse von Automobilität anhand der (Nicht-)Relevanz von ausgesuchten Indikatoren. Autos galten lange Zeit als Ausdruck für einen modernen Lebensstil. Indikatoren zur Analyse ihrer Auswirkung auf Luftqualität und Treibhauseffekt wurden in den 1950er- und 1960er-Jahren in Studien zur Technologieentwicklung nicht herangezogen. Von 1950 bis 1980 nahm die Zahl der Pkws in Wien von 19.216 auf 470.826 zu. Ab 1980, nach der Energiekrise, gab es einen Impuls für effizientere und umweltschonendere Pkws und eine kontextoffene Betrachtung der Mobilität. Dennoch hat das Prestige einer Marke im Pkw-Markt nach wie vor einen hohen Stellenwert in Kaufentscheidungen von Pkws. Indikatoren, wie der langfristige gesamtgesellschaftliche gesundheitliche Schaden, spielen bei Kaufentscheidungen nur eine begrenzte Rolle. Das lässt sich als kontextneutralisierende Operation verstehen.

Für die TA stellt sich dann die Frage, mit welchen Indikatoren sich eine Erweiterung des Blickfeldes in der Praxis erzielen lässt, also solche Formen der Kontextneutralisierung aufgebrochen werden können. Neben der Konturierung einer Problemlage ermöglichen Indikatoren darüber hinaus einen Vergleich zwischen Ist- und Sollwerten, weil sie Differenzsetzungen abbildbar machen. Gerade deshalb stellt die Indikatorenarbeit einen anspruchsvollen Prozess dar, der im Rahmen von TA-Projekten oftmals nicht die gebührende Aufmerksamkeit erhält (Boavida und Böschen 2015). Dabei lassen sich zumindest drei relevante Problemkreise ausmachen.

Der erste Problemkreis hängt mit verschiedenen Definitionen und Nutzungsformen von Indikatoren zusammen. Dabei werden Indikatoren von einer Problemstellung auf eine andere übertragen, obgleich Analogiesicherheit nicht notwendig gegeben ist. Die unterstellte Analogiesicherheit offenbart eine kontextneutralisierende Analysestrategie. Denn dabei werden gleichbleibende Systemvariablen zu dessen Charakterisierung unterstellt, obgleich diese sich ja gerade bei einer problemorientierten Forschung in Abhängigkeit von dem Lösungseingriff sehr wohl unterscheiden können. Die Frage der Repräsentativität von Indikatoren für ein ausgesuchtes Problem muss also immer wieder aufgeworfen werden. Mit Blick auf das Beispiel: Der Indikator „,mobilitätsbedingte Schadstoffbelastung der Luft“ ist z. B. je nach Vorbelastung der Umgebung oder Besiedelungsdichte zu spezifizieren. Eine kontextoffene Strategie ist hierbei behilflich.

Der zweite Problemkreis ergibt sich daraus, dass mit Indikatoren Trugbilder über die zu definierenden und zu lösenden Probleme entstehen können. Bei komplexen Problemen kommt es notwendigerweise zu einem Beschreibungsdefizit durch Indikatoren. Dies ist grundsätzlich auch gar nicht aufzulösen. Daneben stellen sich, viel basaler und zugleich schlichter, oftmals Fragen der Datenverfügbarkeit, oder -aggregation, welche die Auswahl von Indikatoren letztlich steuern. Zudem erzeugt die Festlegung von Systemgrenzen Wirklichkeitsbeschreibungen, welche, bildlich gesprochen, die Differenz von Karte und Gebiet weiter ver- tiefen. Kontextneutralisierende Strategien lassen diese Differenz mehr oder weniger unbeachtet. Bei kontextoffenen Analysestrategien wird dieser Umstand dadurch kompensiert, dass systematisch die Reduktion von Beschreibungskomplexität nachvollziehbar und damit durch die Analyse entstehende Beschreibungsdefizite transparent gemacht werden.

Der dritte Problemkreis schließlich verbindet sich mit der politischen Deutung von Indikatoren. Die Nutzung von Indikatoren suggeriert das Vorliegen von Steuerungsfähigkeit wie -rationalität. Mit Indikatoren werden nicht nur steuerungsfähige Gegenstände geschaffen, sondern durch diese Wissensform eine inhärente Rationalität des Steuerungszwecks mit konstituiert. Zugleich prägen Indikatoren Wirklichkeitsbilder, welche dann als Zielbilder für die gesellschaftliche Entwicklung zirkulieren und einflussreich werden können (wie etwa Wirtschaftsentwicklung durch BIP zu betrachten).

Indikatorenarbeit berücksichtigt gezielt beide Analysestrategien - oder sollte dies zumindest tun. Kontextneutralisierende Analysestrategien beruhen darauf, dass Systeme möglichst mit einem Standardset von Indikatoren behandelt werden. Mittels dieses methodischen Vorgehens wird ein objektiviertes Bild von der Problemsituation und den Lösungsstrategien sowie Modelleindeutigkeit angestrebt. Bei kontextoffenen Analysestrategien hingegen wird die Indikatorenarbeit als Konstruktionsarbeit von Problemen und ihrer Lösung verstanden, bei der die Beschreibungsvielfalt gewürdigt und auf dieser Basis eine pragmatisch wirkungsvolle Problemdeutung vorgenommen wird. In Abhängigkeit von der gesellschaftlichen Problemlösungssituation in einem zur Untersuchung stehenden Feld kann TA die beiden Strategien gezielt einsetzen. Wenn die öffentlich-politische Problemdebatte an problematischen Fokussierungen leidet, dann sollte TA auf kontextoffene Strategien setzen, um den Problemlösungshorizont zu weiten - verliert sich die Debatte hingegen in Unübersichtlichkeit, dann können kontextneutralisierende Angebote zur Komplexitätsreduktion hilfreich sein. Gleichwohl ist immer auf den Gegenstandsbezug zu achten. Dies umso mehr, als Akteure ihre partikulären Perspektiven mit je eigenen Indikatoren gerade dann exponieren, wenn eine übergreifende Strukturierung von Problemen noch nicht vorliegt. Indikatorenarbeit und damit einhergehende Besonderheiten werden nachfolgend an einem TA-Projekt im Feld der Nachhaltigkeit verdeutlicht.

\section{Indikatorenarbeit in einem konkreten TA-Projekt}

Nachhaltigkeitsanalysen, wie jene von Kopfmüller et al. (2001), basieren häufig auf hoch elaborierten Bewertungssystemen, in denen die Indikatorenarbeit relativ stark formalisiert ist. $\mathrm{Zu}$ gleich zeigen sich hier auch die Grenzen der Formalisierung, so dass deutlich gemacht werden kann, wie normative Wertsetzungen in die Analyse einfließen und das Ergebnis prägen. Das konkrete Projektbeispiel stammt aus dem Feld des „Integrativen Konzepts nachhaltiger Entwicklung" (IKoNE) (Kopfmüller et al. 
2001). Es bezieht sich auf einen kleinräumigen Fall, das Konzept kann gleichwohl aber auf beliebig komplizierte Prozesse angewendet werden (Schippl et al. 2017).

\section{IKoNE: Grundlagen}

Die normativen Grundlagen der Nachhaltigkeitsdefinition für IKoNE folgen den drei konstitutiven Elementen der Nachhaltigkeit (Hauff 1987):

- Inter- und intragenerative Gerechtigkeit;

- Betrachtung der lokalen, regionalen und globalen Bezugsrahmen;

- Naturschutz in einem anthropozentrisch begründeten, aber sehr weiten Bedeutungsrahmen.

Diese konstitutiven Elemente werden in IKoNE in drei generelle Ziele übersetzt: (1) die Sicherung der menschlichen Existenz; (2) die Erhaltung des gesellschaftlichen Produktivpotenzials sowie (3) die Bewahrung der Entwicklungs- und Hand-

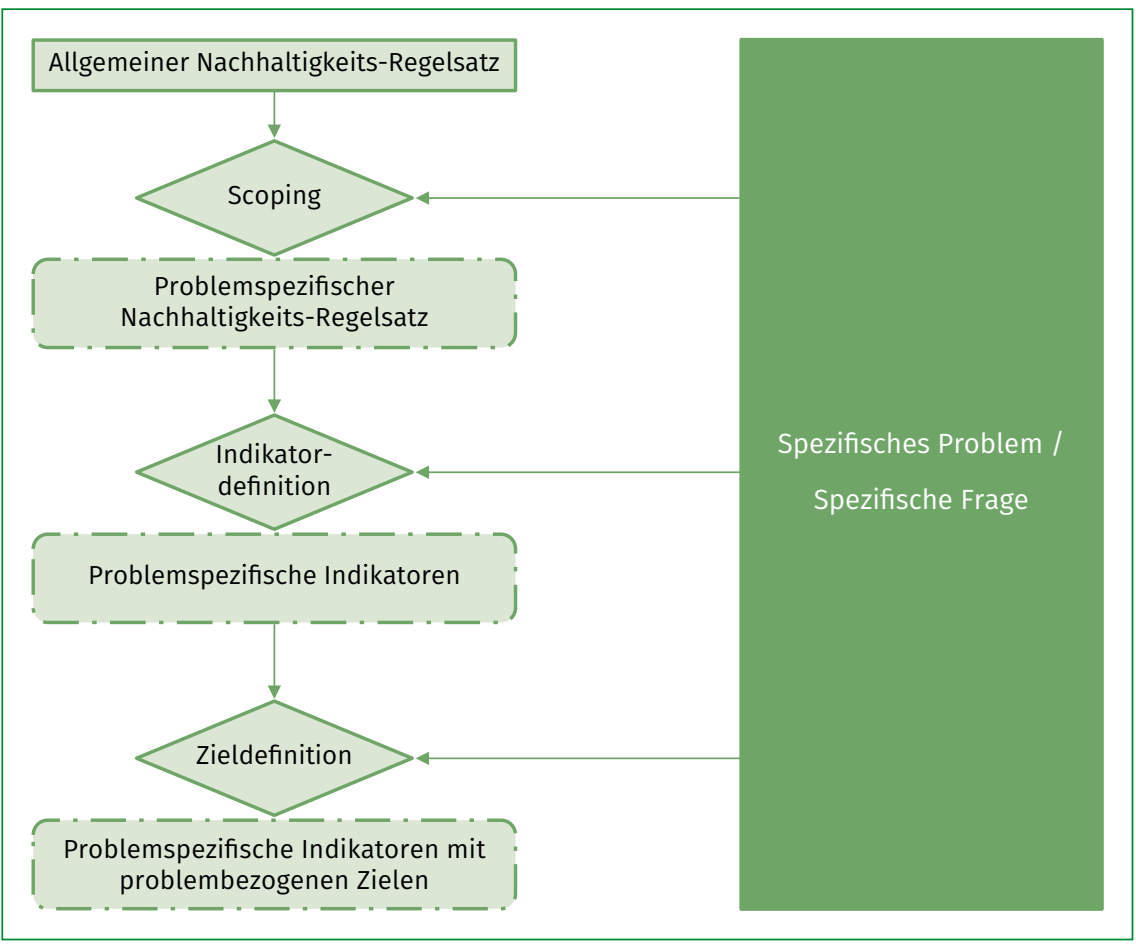
lungsmöglichkeiten der Gesellschaft. Diese stellen die erste Ebene der normativen Hierarchie der Nachhaltigkeitsbewertung nach IKoNE dar. Die drei generellen Nachhaltigkeitsziele werden durch 15 substanzielle Regeln oder Handlungsleitlinien konkretisiert (Tabelle 1). Diese explizit normative Herleitung der Nachhaltigkeitsziele basiert zum einen auf dem Brundtlandbericht und den Rio-Dokumenten, ist zum anderen Ergebnis unterschiedlicher wissenschaftlicher Diskurse. Die Begründung der Entscheidung für die eine oder die andere Definition, z. B. von Gerechtigkeit als soziale Gerechtigkeit (ebd., S. $130 \mathrm{ff}$.), kann so transparent und zum Gegenstand von Diskursen gemacht werden. Dies ist eine normative Setzung im Modell selbst.

Diese Regeln werden generell bei Nachhaltigkeitsbewertungen angewendet. Dabei wird in einem ersten Schritt, dem Scoping, geprüft, ob für die konkrete Fragestellung, wie z. B. die Anwendung einer Technologie in einem bestimmten gesellschaftlichen Kontext, alle Regeln betroffen sind (Abb. 1). Diese Regelauswahl ist direkt abhängig von der Fragestellung, d. h. dem Kontext. Dieser Schritt der Indikatorenarbeit, welcher genau genommen die Kriterien auswählt, zu denen späterhin Indikatoren definiert werden, hat einen erheblichen kontextneutralisierenden Effekt - bei gleichzeitig weitreichender normativer Wertung.

\section{Ländliche Räume in Baden-Württemberg}

Im sogenannten Grünlandprojekt, das von 2003 bis 2006 vom Institut für Technikfolgenabschätzung und Systemanalyse (ITAS) im Auftrag des Ministeriums für Ländlichen Raum und Verbraucherschutz Baden-Württemberg durchgeführt wurde (Rösch

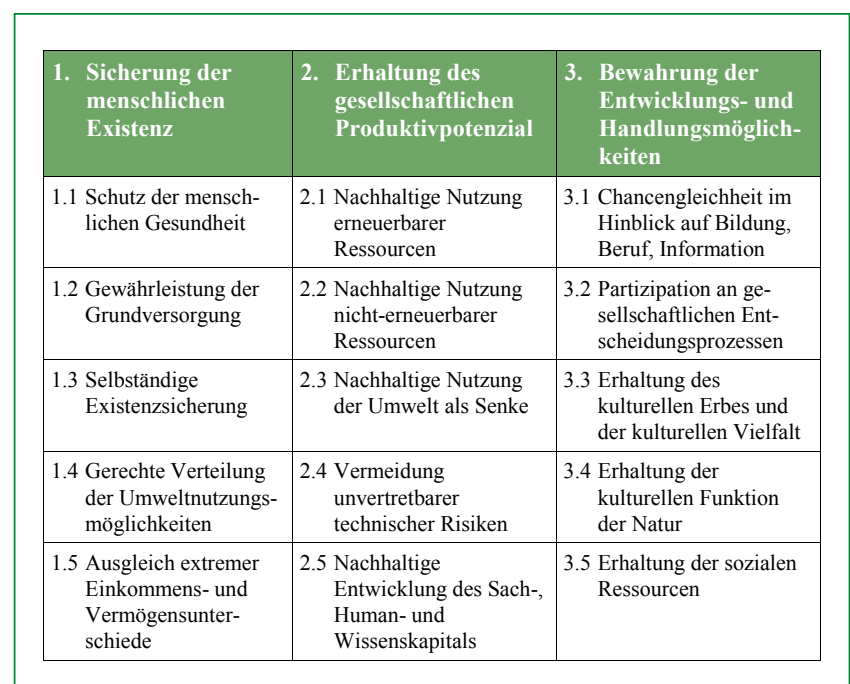

Tab. 1: Die drei generellen Ziele und die ihnen zugeordneten substanziellen Nachhaltigkeitsregeln (die „Was-Regeln“ der Nachhaltigkeit).

Quelle: Kopfmüller et al. 2001, S. 172

2007; Rösch et al. 2007), lautete die Fragestellung: „Ist energetische Nutzung der Grasaufwuchs auf Überschussgrünland nachhaltig?" Um diese Frage zu beantworten, wurden in einem Workshop mit Expert*innen und Stakeholdern die folgenden sieben Regeln als relevant eingeschätzt: (1.1) Der Schutz der menschlichen Gesundheit; (1.3) Selbständige Existenzsicherung; (1.4) 


\begin{tabular}{|c|c|}
\hline $\begin{array}{l}\text { Mindestbedingungen } \\
\text { nachhaltiger Entwicklung }\end{array}$ & Nachhaltigkeitsindikatoren \\
\hline $\begin{array}{l}\text { Gerechte Verteilung der } \\
\text { Umweltnutzungsmöglichkeiten }\end{array}$ & $\begin{array}{l}\text { Substitution nicht erneuerbarer Energieträger } \\
\text { Klimarelevante Emissionen }\end{array}$ \\
\hline $\begin{array}{l}\text { Nachhaltige Nutzung nicht } \\
\text { erneuerbarer Ressourcen }\end{array}$ & $\begin{array}{l}\text { Substitution nicht erneuerbarer Energieträger (s.o.) } \\
\text { Flächenspezifischer Primärenergieertrag } \\
\text { Flächenspezifische Vermeidung klimarelevanter Emissionen }\end{array}$ \\
\hline $\begin{array}{l}\text { Nachhaltige Nutzung der } \\
\text { Umwelt als Senke }\end{array}$ & $\begin{array}{l}\text { Klimarelevante Emissionen (s.o.) } \\
\mathrm{CO}_{2} \text {-Vermeidungskosten } \\
\text { Eutrophierend wirkende Emissionen } \\
\text { Versauernd wirkende Emissionen }\end{array}$ \\
\hline $\begin{array}{l}\text { Schutz der menschlichen } \\
\text { Gesundheit }\end{array}$ & $\begin{array}{l}\text { Feinstaubemissionen } \\
\mathrm{NO}_{\mathrm{x}} \text {-Emissionen } \\
\mathrm{CO}-\text { Emissionen } \\
\text { Sommersmog } \\
\text { Pilzsporen }\end{array}$ \\
\hline $\begin{array}{l}\text { Nachhaltige Nutzung } \\
\text { erneuerbarer Ressourcen }\end{array}$ & $\begin{array}{l}\text { Biodiversität } \\
\text { Boden } \\
\text { Grund- und Oberflächengewässer }\end{array}$ \\
\hline $\begin{array}{l}\text { Erhalt der kulturellen } \\
\text { Funktion der Natur }\end{array}$ & Landschaftsbild \\
\hline $\begin{array}{l}\text { Selbstständige } \\
\text { Existenzsicherung }\end{array}$ & $\begin{array}{l}\text { Beschäftigung } \\
\text { Entlohnung }\end{array}$ \\
\hline
\end{tabular}

Tab. 2: Ausgewählte Indikatoren zur Operationalisierung der Mindestanforderungen nachhaltiger Entwicklung.

Quelle: Rösch et al. 2007, S. 103

beim Erstellen eines Gesamtindikatorensatzes treffen zwei konfligierende pragmatische Ansprüche aufeinander: Je mehr Aspekte einer Regelanwendung durch Indikatoren abgedeckt werden, desto umfassender und damit klarer repräsentiert der Indikatorensatz die Regelanwendung. Aber jeder Indikator mehr erhöht Aufwand wie Kosten und macht die Darstellung potenziell unübersichtlicher. Bei diesem Schritt zeigt sich die schwierige Balance zwischen kontextneutralisierenden und kontextoffenen Analysestrategien ganz prägnant. Neutralisierung erlaubt eine knappe, prägnante und oftmals pragmatisch einfachere Analyse. Öffnung bedeutet Aufwand, manchmal auch Unübersichtlichkeit; dafür geht sie jedoch mit einer detailreicheren Darstellung der Problemlage einher. Da das Ergebnis von derartigen Prozessen oft an externe Akteure (Politik, Unternehmer) gerichtet ist, die nur über begrenzte Aufmerksamkeitsressourcen verfügen, kann sich leicht eine

Gerechte Verteilung der Umweltnutzungsmöglichkeiten; (2.1) Nachhaltige Nutzung erneuerbarer Ressourcen; (2.2) Nachhaltige Nutzung nicht erneuerbarer Ressourcen; (2.3) Nachhaltige Nutzung der Umwelt als Senke sowie (3.4) Erhalt der kulturellen Funktion der Natur. Diese Regeln wurden in einem nächsten Schritt durch Indikatoren operationalisiert (Abb. 1). Der prinzipiell kontextneutralisierenden Qualität dieses Schrittes mit der Gefahr implizit bleibender normativer Wertungen kann durch Perspektivendifferenzierung in Beteiligungsverfahren entgegengewirkt werden.

Die Gesundheitsregel (1.1) wurde vor allem mit den Indikatoren $\mathrm{NO}_{\mathrm{x}}$ - und $\mathrm{CO}$-Emissionen bewertet, da diese Substanzen bei der Umwandlung von Grünlandaufwuchs in Energie frei-
Präferenz für kontextneutralisierende Strategien einschleichen. Auch hier war ein mehrschichtiger Diskurs mit ExpertInnen die Methode der Wahl, wobei 18 Indikatoren herausgearbeitet wurden (Tabelle 2). Bei aller methodischer Finesse spiegelt ein solcher Indikatorensatz freilich normative Setzungen wider.

Für einen Teil der Indikatoren wurden anschließend Stoffstromanalysen erstellt (z. B. klimarelevante Emissionen und $\mathrm{NO}_{\mathrm{x}}$-Emissionen durch Biomasseverbrennung). Für andere wurden quantitative Berechnungen durchgeführt (Beschäftigung, Entlohnung), und einige wurden verbal beschrieben (z. B. Biodiversität). Für die Betrachtungen erfolgten Festlegungen von Kontext (Bilanzgrenzen) und von Rahmenbedingungen, die bei der Erarbeitung der Indikatorwerte herangezogen wurden. Die

\section{Für die Zusammenstellung eines Indikatorensatzes gilt: „So viel Indikatoren wie nötig, aber so wenig wie möglich“.}

gesetzt werden können. Gesundheitsschäden durch radioaktive Strahlung wurden hingegen nicht betrachtet, da diese im genannten Fall keine Rolle spielen. Neben sachlichen Kriterien spielen ebenso pragmatische Kriterien wie die Datenverfügbarkeit oder -qualität eine Rolle. Wenn es zu einem Indikator keine Angaben - Zahlenwerte oder deskriptive Beschreibungen - gibt, so kann dieser nicht für die Beantwortung der vorliegenden Fragestellung herangezogen werden.

Für die Zusammenstellung eines Indikatorensatzes gilt: ,So viel Indikatoren wie nötig, aber so wenig wie möglich“. Denn
Festlegung der Bilanzgrenzen ist ein Schritt, bei dem ebenfalls normative Setzungen vorgenommen werden (z. B. ob der Energieaufwand bei der Kunstdüngerproduktion in die Bewertung einbezogen wird, oder, ob das Ausbringen der Reste aus der Biogasanlage auf das Feld Berücksichtigung findet, oder nicht). Schließlich enthalten die Zielwerte, die erreicht werden sollen, spezifische normative Festlegungen, die oftmals durch die geltenden Gesetze vorgegeben werden - aber in Studien, die nicht auf solche rechtlichen Festlegungen zurückgreifen können, spiegeln Zielwerte Erwartungen wider, wobei darauf geachtet wer- 
den muss, dass dies nicht selektiv Erwartungen partikularer Akteursgruppen sind.

Tabelle 3 zeigt, dass mit Hilfe von Indikatoren methodisch verschieden generierte Fakten in einem vereinheitlichten Bewertungsschema und Modell dargestellt wurden. Diese Tabelle macht deutlich, dass ein solches Ergebnis, das eine Fülle von kontextoffenen Elementen enthält (Vielzahl von Kriterien und Indikatoren einerseits sowie Vielfalt von Handlungsoptionen in Form von Varianten der Landwirtschaft wie Technologien andererseits), zwar eine vielschichtigere Beschreibung erlaubt, zugleich aber Entscheidungskomplexität nur begrenzt reduziert. Auf der anderen Seite macht sie aber die konkreten Entscheidungsmöglichkeiten und ihre normativen Bezüge transparent.

In dem beschriebenen Fall realisierte sich die kontextbezogene Normativität demnach insbesondere an vier Stellen im Bewertungsprozess: 1) Bei der Auswahl der als relevant eingeordneten Regeln; 2) der Festlegung der Bilanzgrenzen; 3) der problemorientierten Auswahl der Indikatoren; und schließlich 4) der Festlegung der Bewertungsmaßstäbe (Zielwerte, Referenzwerte etc.).

\section{Diskussion und Ausblick}

Das geschilderte Projekt zeigt auf, wie in allen Schritten Indikatorenarbeit als Konfiguration von kontextneutralisierenden und kontextoffenen Analysestrategien geleistet werden muss. Auf der einen Seite werden Indikatoren in Nachhaltigkeitsanalysen durch regelbasiertes Vorgehen schrittweise begrenzt und definiert. Auf diese Weise wird kontextneutralisierend der Raum der Beschreibung so weit reduziert, dass ein Problembinnenraum entsteht, der dann Gegenstand der Analyse ist. Auf der anderen Seite öffnet sich in jeder Phase des Drei-Schritts vom Scoping über die Indikatordefinition zur Zieldefinition zugleich ein Gelegenheitsfenster für eine kontextoffene Arbeitsstrategie. Indem nämlich die jeweiligen Selektionsprozesse reflexiv vollzogen werden, kann der Außenraum (Kontext) im Problembinnenraum immer wieder zum Sprechen gebracht werden.

Ein zentraler Punkt, der für den Ausgleich der inhärenten Spannung zwischen einer kontextneutralisierenden und kontext-

\begin{tabular}{|c|c|c|c|c|c|c|c|c|c|}
\hline & \multicolumn{5}{|c|}{ Extensive Grünlandflächen } & \multicolumn{4}{|c|}{ Intensive Grünlandflächen } \\
\hline & \multicolumn{4}{|c|}{ Nutzung } & \multirow{2}{*}{ 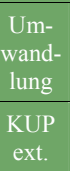 } & \multirow{2}{*}{$\begin{array}{l}\begin{array}{l}\text { Nut- } \\
\text { zung }\end{array} \\
\begin{array}{l}\text { Gras- } \\
\text { silage }\end{array}\end{array}$} & \multicolumn{3}{|c|}{ Umwandlung } \\
\hline & $\begin{array}{l}\mathrm{Heu} \\
\mathrm{HD}\end{array}$ & $\begin{array}{l}\mathrm{Heu} \\
\mathrm{RB}\end{array}$ & $\begin{array}{l}\text { Heu } \\
\text { Pellets }\end{array}$ & $\begin{array}{l}\text { Heu } \\
\mathrm{TF}^{1)}\end{array}$ & & & $\begin{array}{l}\text { Mais- } \\
\text { silage }\end{array}$ & $\begin{array}{l}\text { KUP } \\
\text { int. }\end{array}$ & $\mathrm{KUP}^{2)}$ \\
\hline \multicolumn{10}{|c|}{ Nachhaltige Nutzung nicht erneuerbarer Ressourcen } \\
\hline Primärenergieertrag & $\begin{array}{l}++ \\
(7)^{3)} \\
\end{array}$ & $\begin{array}{l}++ \\
(6)\end{array}$ & $\begin{array}{l}++ \\
(5)\end{array}$ & $\begin{array}{l}++ \\
(8)\end{array}$ & $\begin{array}{l}++ \\
(3)\end{array}$ & $\begin{array}{l}++ \\
(4) \\
\end{array}$ & $\begin{array}{l}++ \\
(2)\end{array}$ & $\begin{array}{l}++ \\
(1)\end{array}$ & $\begin{array}{l}++ \\
(1)\end{array}$ \\
\hline \multicolumn{10}{|c|}{ Nachhaltige Nutzung der Umwelt als Senke } \\
\hline $\begin{array}{l}\text { Klimarelevante } \\
\text { Emissionen }\end{array}$ & $\begin{array}{l}++ \\
(6)\end{array}$ & $\begin{array}{l}++ \\
(5)\end{array}$ & $\begin{array}{l}++ \\
(4)\end{array}$ & $\begin{array}{c}+ \\
+ \\
(8)\end{array}$ & $\begin{array}{l}++ \\
(2)\end{array}$ & $\begin{array}{l}++ \\
(7)\end{array}$ & $\begin{array}{l}++ \\
(3)\end{array}$ & $\begin{array}{l}++ \\
(1)\end{array}$ & $\begin{array}{l}++ \\
(1)\end{array}$ \\
\hline $\begin{array}{l}\mathrm{CO}_{2} \text {-Vermeidungs- } \\
\text { kosten }\end{array}$ & $\begin{array}{l}- \\
(5)\end{array}$ & (3) & (3) & $\begin{array}{l}-- \\
(6)\end{array}$ & $\begin{array}{c}+ \\
+ \\
(2)\end{array}$ & $\begin{array}{l}-- \\
(4)\end{array}$ & $\begin{array}{l}-- \\
(4)\end{array}$ & $\begin{array}{l}++ \\
(1)\end{array}$ & $\begin{array}{l}++ \\
(1)\end{array}$ \\
\hline $\begin{array}{l}\text { Eutrophierende } \\
\text { Emissionen }\end{array}$ & $\begin{array}{l}- \\
(5)\end{array}$ & $\begin{array}{c}0 \\
(3)\end{array}$ & $\begin{array}{l}- \\
(6)\end{array}$ & $\begin{array}{l}-- \\
(9)\end{array}$ & $\begin{array}{c}0 \\
(2)\end{array}$ & $\begin{array}{l}-- \\
(8)\end{array}$ & $\begin{array}{l}-- \\
(7)\end{array}$ & $\begin{array}{c}0 \\
(3)\end{array}$ & $\begin{array}{c}0 \\
(1)\end{array}$ \\
\hline $\begin{array}{l}\text { Versauernde } \\
\text { Emissionen }\end{array}$ & $\overline{-}$ & $\begin{array}{c}0 \\
(4) \\
\end{array}$ & $\begin{array}{l}- \\
(6)\end{array}$ & $\begin{array}{l}- \\
(9)\end{array}$ & $\begin{array}{c}+ \\
(3) \\
\end{array}$ & $\begin{array}{l}-- \\
(8)\end{array}$ & $\begin{array}{l}-- \\
(7)\end{array}$ & $\begin{array}{c}+ \\
(2) \\
\end{array}$ & $\begin{array}{c}+ \\
(1) \\
\end{array}$ \\
\hline \multicolumn{10}{|c|}{ Schutz der menschlichen Gesundheit } \\
\hline Feinstaubemissionen & $\begin{array}{c}0 \\
(4)\end{array}$ & $\begin{array}{c}-\overline{1} \\
(7) \\
\end{array}$ & $\begin{array}{c}- \\
(5) \\
\end{array}$ & $\begin{array}{c}+ \\
(1) \\
\end{array}$ & $\begin{array}{c}- \\
(8)\end{array}$ & $\begin{array}{c}0 \\
(2) \\
\end{array}$ & $\begin{array}{c}0 \\
(3) \\
\end{array}$ & $\begin{array}{l}-- \\
(9)\end{array}$ & $\begin{array}{c}- \\
(6) \\
\end{array}$ \\
\hline $\mathrm{NO}_{\mathrm{x}}$-Emissionen & $\begin{array}{l}-- \\
(7)\end{array}$ & $\begin{array}{l}-- \\
(6) \\
\end{array}$ & $\begin{array}{l}-- \\
(8)\end{array}$ & $\begin{array}{c}+ \\
(1) \\
\end{array}$ & $\begin{array}{c}- \\
(4)\end{array}$ & $\begin{array}{l}- \\
(3) \\
\end{array}$ & $\begin{array}{l}- \\
(5) \\
\end{array}$ & (6) & $\begin{array}{l}- \\
(2)\end{array}$ \\
\hline CO-Emissionen & $\begin{array}{l}-- \\
(9)\end{array}$ & $\begin{array}{c}+ \\
(2) \\
\end{array}$ & $\begin{array}{c}0 \\
(3)\end{array}$ & $\begin{array}{c}0 \\
(4)\end{array}$ & $\begin{array}{l}- \\
(6)\end{array}$ & $\begin{array}{l}-\overline{1} \\
(5) \\
\end{array}$ & $\begin{array}{l}-- \\
(7)\end{array}$ & $\begin{array}{l}-- \\
(8 \\
\end{array}$ & $\begin{array}{c}+ \\
(1) \\
\end{array}$ \\
\hline Sommersmog & $\begin{array}{l}-- \\
(8)\end{array}$ & $\begin{array}{l}- \\
(7)\end{array}$ & $\begin{array}{l}-- \\
(9)\end{array}$ & $\begin{array}{c}+ \\
(1)\end{array}$ & $\begin{array}{l}- \\
(4)\end{array}$ & (3) & $\begin{array}{l}- \\
(5)\end{array}$ & $\begin{array}{l}- \\
(6)\end{array}$ & $\begin{array}{c}0 \\
(2)\end{array}$ \\
\hline Pilzsporen & 0 & 0 & 0 & - & - & 0 & 0 & - & - \\
\hline \multicolumn{10}{|c|}{ Nachhaltige Nutzung erneuerbarer Ressourcen } \\
\hline Biodiversität & $\begin{array}{c}+ \\
(1) \\
\end{array}$ & $\begin{array}{c}+ \\
(1) \\
\end{array}$ & $\begin{array}{l}+ \\
\text { (1) } \\
\end{array}$ & $\begin{array}{c}+ \\
(1) \\
\end{array}$ & $\begin{array}{l}- \\
(4)\end{array}$ & $\begin{array}{l}0 /- \\
(2)\end{array}$ & $\begin{array}{l}-- \\
(5)\end{array}$ & $\begin{array}{c}0 \\
(3)\end{array}$ & $\begin{array}{c}0 \\
(3) \\
\end{array}$ \\
\hline Boden & $\begin{array}{c}0 \\
(1)\end{array}$ & $\begin{array}{c}0 \\
(1)\end{array}$ & $\begin{array}{c}0 \\
(1)\end{array}$ & $\begin{array}{c}0 \\
(1) \\
\end{array}$ & $\begin{array}{l}- \\
(2)\end{array}$ & $\begin{array}{c}0 \\
(1) \\
\end{array}$ & $\begin{array}{l}-- \\
(3)\end{array}$ & $\begin{array}{l}- \\
(2)\end{array}$ & $\begin{array}{l}- \\
(2) \\
\end{array}$ \\
\hline $\begin{array}{l}\text { Grund- und Oberflä- } \\
\text { chengewässer }\end{array}$ & $\begin{array}{c}0 \\
(1)\end{array}$ & $\begin{array}{c}0 \\
(1)\end{array}$ & $\begin{array}{c}0 \\
(1)\end{array}$ & $\begin{array}{c}0 \\
(1)\end{array}$ & $\begin{array}{l}- \\
(2)\end{array}$ & $\begin{array}{c}0 \\
(1)\end{array}$ & $\begin{array}{l}-- \\
(3)\end{array}$ & $\begin{array}{l}- \\
(2)\end{array}$ & $\begin{array}{l}- \\
(2)\end{array}$ \\
\hline \multicolumn{10}{|c|}{ Kulturelle Funktion der Natur } \\
\hline Landschaft & $\begin{array}{c}+ \\
\text { (1) }\end{array}$ & $\begin{array}{c}+ \\
\text { (1) }\end{array}$ & $\begin{array}{c}+ \\
\text { (1) }\end{array}$ & $\begin{array}{c}+ \\
(1)\end{array}$ & $\begin{array}{l}-/+ \\
(2)\end{array}$ & $\begin{array}{c}+ \\
\text { (1) }\end{array}$ & $\begin{array}{l}- \\
\text { (3) }\end{array}$ & $\begin{array}{l}-1+ \\
(2)\end{array}$ & $\begin{array}{l}-1+ \\
(2)\end{array}$ \\
\hline \multicolumn{10}{|c|}{ Selbstständige Existenzsicherung } \\
\hline Beschäftigung & $\begin{array}{c}+ \\
(1)\end{array}$ & $\begin{array}{c}+ \\
\text { (4) }\end{array}$ & $\begin{array}{c}+ \\
(8) \\
\end{array}$ & $\begin{array}{c}+ \\
(7)^{4)}\end{array}$ & $\begin{array}{l}+ \\
(6)\end{array}$ & $\begin{array}{c}+ \\
(5) \\
\end{array}$ & $\begin{array}{c}+ \\
(2)\end{array}$ & $\begin{array}{c}+ \\
(3)\end{array}$ & $\begin{array}{c}+ \\
(3) \\
\end{array}$ \\
\hline Entlohnung & $\begin{array}{c}- \\
(7) \\
\end{array}$ & $\begin{array}{c}+ \\
(5) \\
\end{array}$ & $\begin{array}{l}- \\
(8)\end{array}$ & $\begin{array}{l}+ \\
(6)^{4)} \\
\end{array}$ & $\begin{array}{l}++ \\
(2)\end{array}$ & $\begin{array}{c}+ \\
(4) \\
\end{array}$ & $\begin{array}{c}+ \\
(3) \\
\end{array}$ & $\begin{array}{l}++ \\
(1)\end{array}$ & $\begin{array}{l}++ \\
(1)\end{array}$ \\
\hline
\end{tabular}

${ }^{1)}$ Trockenfermentation von Heu zusammen mit Maissilage.

${ }^{2)}$ Verbrennung von Kurzumtriebspappeln (KUP) in Kesseln mit emissionsarmer Verbrennung.

3) +/- geben an, ob das Verfahren im Vergleich zur Referenz (Mulchen, fossile Energieträger) positiv/negativ

ist, die Zahlen 1 (gut) bis 9 (schlecht) die Rangfolge der Verfahren verglichen mit den anderen Verfahren.

${ }^{4)}$ Diese Angaben gelten für die Co-Fermentation von Extensivheu mit Maissilage.

Tab. 3: Bewertung der unterschiedlichen Verfahren. Obwohl einige Indikatoren durch Modelle oder eigene Tools berechnet oder verbal beschrieben wurden, wurden sie in diesem Fall durch eine fünfstufige Skala $(++,+, 0,-,--)$ normiert. Quelle: Rösch et al. 2007, S. 144 offenen Analysestrategie ins Auge sticht, ist die soziale Absicherung der Prozessschritte - mit zwei Aspekten. Zum einen kann die sachliche Komplexität umso eher hinsichtlich ihrer normativen Beeinflussung erfasst werden, wenn es gelingt, durch eine korrespondierende soziale Prozesskomplexität die Chancen auf Transparenz zu steigern. Entscheidend ist also eine durch Indikatoren gestützte Bewertung so durchzuführen, dass die Kriterien für die Indikatorenauswahl transparent und nachvollzieh- 
bar sind. Zum anderen kann diese Analyse umso eher gelingen, wenn durch den Einbezug von unterschiedlichen Akteuren der Kontext genauer zum Sprechen gebracht wird und zu Beginn jedes Schrittes Beteiligungsverfahren eingesetzt werden, um die Umwelt im sukzessive enger definierten Problembinnenraum angemessen zu repräsentieren und dafür die entsprechenden Messgrößen zu sichern. Mit dieser Doppelstrategie (sachlich und sozial) kann die Normativität von TA-Analysen in ihrer Kontextbezogenheit transparent gemacht werden.

Dies ist umso bedeutsamer als die Zunahme von Komplexität im Betrachtungsgegenstand der TA (etwa: Einzeltechnologie, Technologiefamilie, Technologie in ihrem Anwendungsfeld, Region mit den angewandten Technologien) mit einer $\mathrm{Zu}$ nahme normativer Setzungen einhergeht, die in der Regel im Rahmen der Bewertung durchgeführt werden. Zugleich verbinden sich mit diesen Setzungen unterschiedliche Entwicklungsperspektiven, so dass hier ein Feld unterschiedlicher Ausgangsbezüge und Entwicklungsperspektiven dargestellt werden muss. Denn die Definition des Problembinnenraums stellt bei unstrukturierten Problemen (wie etwa der Energiewende oder dem Klimawandel), bei denen Wissen und Werte umstritten sind, Teil des öffentlich-politischen Prozesses dar.

Die Anwendung von Modellen (als kontextneutralisierende Strategie) funktioniert nur bei strukturierten Problemen (Wissen und Werte sind nicht umstritten), da hier schon ein institutioneller Rahmen zur Problembearbeitung existiert. Ansonsten verknüpfen sich mit kontextneutralisierenden Strategien spezifische Wertsetzungen, die aufgrund der scheinbaren Neutralität von Modellen nicht immer sichtbar sind. Würde man also bei unstrukturierten Problemen voreilig einen Problembinnenraum festlegen, so würde dies dem Ergebnis des öffentlich-politischen Diskurses vorgreifen. Indikatorenarbeit ginge dabei in Indikatorenpolitik über (Böschen 2019).

Offenkundig sind kontextneutralisierende wie kontextoffene Analysestrategien relevant für TA. Entscheidend ist für TA-Praktiker die Einsicht in die Bedingungen der Anwendbarkeit der einen oder anderen Form. Indikatorenarbeit bedeutet eine reflektierte Nutzung beider Analysestrategien, um sachadäquat und normtransparent zu verfahren.

\section{Literatur}

Bechmann, Gotthard (1993): Sozialwissenschaftliche Forschung und Technikfolgenabschätzung. In: Klaus Lompe (Hg.): Techniktheorie - Technikforschung - Technikgestaltung. Opladen: Westdeutscher Verlag, S. 28-58. Boavida, Nuno; Böschen, Stefan (2015): Indicators in technology assessment. Passive choices or reflected options. In: Tomas Michalek et al. (Hg.): The next horizon of technology assessment. Prag: Informatorium, S.33-40.

Böschen, Stefan (2019): TA at the crossroads. Politics of TA from the viewpoint of societal problem solving. In: Technological Forecasting and Social Change 139, S. 42-47.

Bröchler, Stephan; Simonis, Georg; Sundermann, Karsten (Hg.) (1999): Handbuch Technikfolgenabschätzung. Berlin: edition sigma.

Giegel, Hans-Joachim (1993): Kontextneutralisierung und Kontextoffenheit als Strukturbedingungen der gesellschaftlichen Risikokommunikation. In:
Wolfgang Bonss, Rainer Hohlfeld und Regine Kollek (Hg.): Wissenschaft als Kontext. Kontexte der Wissenschaft. Hamburg: Junius, S. 103-121.

Grunwald, Armin (Hg.) (1999): Rationale Technikfolgenbeurteilung. Konzepte und methodische Grundlagen. Berlin: Springer.

Hauff, Volker (Hg.) (1987): Unsere gemeinsame Zukunft. Der BrundtlandBericht der Weltkommission für Umwelt und Entwicklung. Greven: Eggenkamp.

Kopfmüller, Jürgen et al. (2001): Nachhaltige Entwicklung integrativ betrachtet. Konstitutive Elemente, Regeln, Indikatoren. Berlin: edition sigma.

Rösch, Christine; Raab, Konrad; Skarka, Johannes; Stelzer, Volker (2007): Energie aus dem Grünland. Eine nachhaltige Entwicklung?. Karlsruhe: Forschungszentrum Karlsruhe. Online verfügbar unter http://www.itas.kit.edu/pub/v/2007/roua07b.pdf, zuletzt geprüft am 29. 01.2019.

Rösch, Christine (2007): Die Nutzung von überschüssigem Grünland als Energieressource. In: TATuP - Zeitschrift für Technikfolgenabschätzung in Theorie und Praxis 16 (3), S. 88-93.

Schippl, Jens; Grunwald, Armin; Renn, Ortwin (2017): Die Energiewende verstehen orientieren - gestalten. Einsichten aus fünf Jahren integrativer Forschung. In: Jens Schippl, Armin Grunwald und Ortwin Renn (Hg.): Die Energiewende verstehen - orientieren - gestalten. Erkenntnisse aus der Helmholtz-Allianz ENERGY-TRANS. Baden-Baden: Nomos, S. 9-34.

Stelzer, Volker; Kopfmüller, Jürgen (2019, im Erscheinen): Sustainability assessment. Integrative concept, methodology and examples. In: Claudia Binder und Stefanie Krauth (Hg.): Sustainability assessments of urban systems. München: Elsevier.

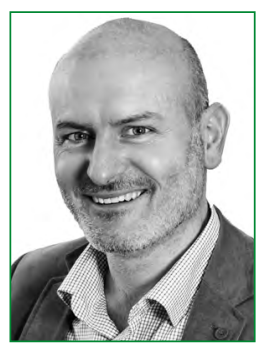

PROF.DR. PHIL. DIPL.-ING. STEFAN BÖSCHEN

leitet den Lehrstuhl „Technik und Gesellschaft“ am Human Technology Center (HumTec) der RWTH Aachen University. Er ist Chemie-Ingenieur und Soziologe mit Schwerpunkten in der Wissenschaftsund Technikforschung sowie Technikfolgenabschätzung.

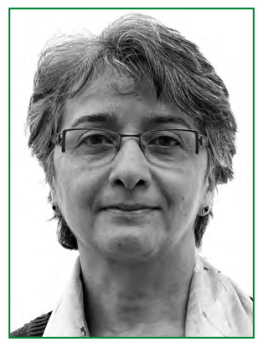

PRIV. DOZ. DI DR.IN MAHSHID SOTOUDEH ist TA-Forscherin mit einem Hintergrund in der Chemie- und Verfahrenstechnik. Ihre Forschungsgebiete sind Technologie und Nachhaltigkeit, partizipative Methoden und Foresight. Sie ist Dozentin mit Venia Legendi „Technikfolgenabschätzung und Nachhaltigkeit" an der Technischen Universität Graz.

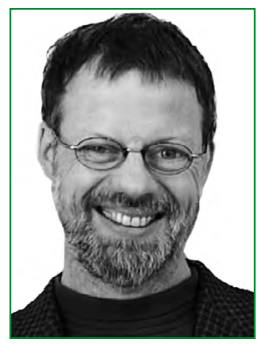

\section{DR. VOLKER STELZER}

ist Senior Scientist und Nachhaltigkeitsforscher am Institut für Technikfolgenabschätzung und Systemanalyse (ITAS) des KIT. Seine Forschungsschwerpunkte sind systematische Nachhaltigkeitsbewertungen in der Stadtentwicklung und der Energieversorgung sowie Zukunftsbetrachtungen mittels Szenarien. 